Article

\title{
Synthesis of Hydrogel Films Based on PVA, PVP, Starch and Keratin extracted from chicken feathers wastes for the Potential biomedical applications
}

\author{
Mohamed Saad Bala Husain1, Basma Yahya Alashwal ${ }^{2}$ Arun Gupta ${ }^{3, *}$, Swati Sharma ${ }^{4}$, Jayarama Reddy Venugopal 5,*, \\ Husam Eldin Elhag Abugabr Elhag ${ }^{6}$, and Triveni Soubam ${ }^{7}$ \\ 1 Faculty of Chemical and Process Engineering Technology, Universiti Malaysia Pahang, 26300, Gambang, \\ Pahang, Malaysia; mohamedsaadbala@gmail.com \\ 2 Faculty of Chemical and Process Engineering Technology, Universiti Malaysia Pahang, 26300, Gambang, \\ Pahang, Malaysia; chem.basma22@gmail.com \\ 3 Faculty of Chemical and Process Engineering Technology, Universiti Malaysia Pahang, 26300, Gambang, \\ Pahang, Malaysia; arun@ump.edu.my \\ 4 University Institute of Biotechnology (UIBT), Chandigarh University, Mohali, Punjab, 140413, India; \\ sspandit.89@gmail.com \\ 5 Faculty of Industrial Sciences \& Technology, Universiti Malaysia Pahang, 26300, Gambang, Pahang, Malay- \\ sia; venugopal@ump.edu.my \\ 6 College of Engineering Technology, Universiti Malaysia Pahang, 26300, Gambang, Pahang, Malaysia; husa- \\ mabugabr@gmail.com \\ 7 Faculty of Chemical and Process Engineering Technology, Universiti Malaysia Pahang, 26300, Gambang, \\ Pahang, Malaysia; triveni23novsoubam@gmail.com \\ *3,5Correspondence: arun@ump.edu.my; venugopal@ump.edu.my
}

\begin{abstract}
The chicken feather wastes are primarily composed of keratin protein, which can be exploited to produce products for biomedical applications. In this research, keratin was extracted from chicken feathers waste and was applied to prepare the hydrogel films for biomedical applications. Hydrogel films, prepared by polyvinyl alcohol (PVA) and polyvinylpyrrolidone (PVP) and corn starch are used at temperature $-20^{\circ} \mathrm{C}$. The effect of keratin in hydrogel films was examined by Fourier-transform infrared spectroscopy (FTIR), confirmed the presence of keratin, scanning electron microscope (SEM) examined surface morphology, the porosity of the hydrogel decreased for KS-70 at $33.57 \%$, due to their relatively high Interconnecting and low porous structure due to their low water content with high keratin content. The swelling ratio of KS70 at $30.66 \%$ after $1440 \mathrm{~min}$ due to its relatively increased crosslinking density with high keratin content. On the other hand, tensile strength was seen improvement with the increase of the keratin protein content into hydrogel films. Moreover, keratin release increased with increasing the keratin content; The Higuchi square root was the optimal model of keratin kinetics release for all the hydrogel films. These results were indicated that feather keratin could use with formulated hydrogels suitably for controlled keratin release studies.
\end{abstract}

Keywords: Hydrogel; keratin; chicken feather waste.

\section{Introduction}

Hydrogels are three-dimensional polymer nets that can absorb large amounts of water by various natural and synthetic polymers [1]. Hydrogels have revolutionized the approaches on the modern wound dressing and drug delivery systems, which they could allow oxygen to permeate, absorb tissue exudates, and prevent wound dehydration and create better healing conditions and controlled release of drug [2,3]. Generally, the hydrogels are formulated by physical or chemical crosslinking using hydrophilic polymers and water-soluble [4,5]. The hydrogels have been used to the controlled release behaviour of protein as well as wound healing applications [6]. 
Keratins are proteins that can be extracted from low-cost sources such as feathers, human hair, and wool [7]. keratins have been widely used to develop in wound healing applications owing to their special properties, such as biocompatibility and biodegradability $[8,9]$. The major component $(>90 \%)$ of chicken feathers is keratin protein rich in cysteine and hydrophobic residues, which facilitates crosslinking by disulphide bonds [10].

Among all the most commonly used polymers to prepare Hydrogels, Polyvinyl alcohol (PVA) has various useful characteristics such as excellent biocompatibility, non-toxicity, high hydrophilicity, good biodegradability, hydrogel film-forming ability, and proper mechanical characteristics [11]. Poly-vinylpyrrolidone (PVP) is also a biodegradable and one of the most commonly water-soluble and non-toxic synthetic polymers [12]. In addition, PVA/PVP-based hydrogels have been described as the potential biomaterials and appropriate candidates for biomedical applications $[13,14]$.

Starch is a natural polymer that possesses abundant distinctive characteristics and one of the most abundantly and the cheapest biodegradable polymers. Starch-based polymers have been suggested as a material for a broad range of biomedical and pharmaceutical applications [15].

In this research paper, hydrogel samples with keratin, PVA, PVP, and starch were formed, characterized, and evaluated keratin release ratio and improved physical, mechanical properties to use for biomedical applications.

\section{Materials and Methods}

The white chicken feathers have been taken from Poultry Farm Sdn. Bhd. Kuantan, Malaysia, Dimethyl sulfoxide (DMSO, 99.9\% purity), polyvinylpyrrolidone powder (PVP), polyvinyl alcohol pellet PVA), and corn starch, were purchased from Sigma-Aldrich.

\subsection{The extraction process of Keratin}

The chicken feather $(100 \mathrm{~g})$ was solubilized by $1 \mathrm{M}$ Sodium hydroxide $(\mathrm{NaOH})$ at $60^{\circ} \mathrm{C}$ with Stirring carried on for $4 \mathrm{~h}$. The resulting mixture was centrifuged at $10000 \mathrm{rpm}$ and $25^{\circ} \mathrm{C}$ for $10 \mathrm{~min}$ to a separate the insoluble material, and the supernatant was filtered through filtering paper to make it particles-free this process was repeated for 3 times as well $\mathrm{pH}$ was then adjusted between 6.8 and 7.2 by dropwise additions of further $\mathrm{HCl}(2 \mathrm{M})$ to the solution. Afterwards, the pure keratin as the powder was obtained through freezedrying [16].

\subsection{Preparation of Polyvinylpyrrolidone (PVP/PVP) Solution}

PVA (12g) was dissolved by $80 \mathrm{~mL}$ DMSO aqueous solution into a glass beaker and heated to $98^{\circ} \mathrm{C}$ with stirring for $3 \mathrm{~h}$. Then, PVP (8g) was added to the PVA/DMSO mixture solution separately with stirring for $2 \mathrm{~h}$ at the same temperature [17].

\subsection{The preparation hydrogel samples}

The keratin solution was prepared by mixing $5 \mathrm{~g}$ of keratin powder into $100 \mathrm{ml}$ of $\mathrm{dH}_{2} \mathrm{O}$ and stirred at $50^{\circ} \mathrm{C}$ for $15 \mathrm{~min}$. The KS-hydrogels were made by mixing keratin, PVA/PVP solution, and $2 \mathrm{~g}$ of starch using the freeze-thaw method as given in Table 3 . The mixtures were heated to $60^{\circ} \mathrm{C}$, and $\mathrm{pH}$ was maintained at 7 . The solution was continuously stirred for $30 \mathrm{~min}$ and then were poured into a petri-dish. The mixture was exposed to three cycles of freezing at $-20^{\circ} \mathrm{C}$ and $8 \mathrm{hr}$ and thawing at $25^{\circ} \mathrm{C}$ for $4 \mathrm{~h}$ to form the KS-hydrogels [18].

.Table 1. Formulation of Hydrogel.

\begin{tabular}{ccc}
\hline Formulation Code & Keratin protein (ml) & PVA/PVP (ml) \\
KS 30 & 30 & 70 \\
KS 40 & 40 & 60 \\
KS 50 & 50 & 50 \\
KS 60 & 60 & 40 \\
KS 70 & 70 & 30 \\
\hline
\end{tabular}




\subsection{Physicochemical characterization of hydrogel samples}

\subsubsection{FTIR}

FTIR was applied to provide information about the chemical composition of the hydrogel samples. The spectrum scopes of hydrogels the spectra were at $4000 \mathrm{~cm}^{-1}$ to 500 $\mathrm{cm}^{-1}$. The results of the FTIR analysis were drawn using OriginPro2017

\subsubsection{SEM}

The morphologies and structures of the hydrogel samples were analyzed by scanning electron microscopy (SEM, FEI Quanta 450). The hydrogel samples were placed on double-sided carbon tape. The micrographs of samples were recorded at $800 \times$ magnification under a scanning electron microscope.

\subsubsection{Porosity Measurements of Hydrogel}

The porosity measurement of hydrogels was determined according to the technique of the solvent replacement. The hydrogel samples were cut to small pieces with $2 \mathrm{~cm} \times 2 \mathrm{~cm}$ square, and they were weighed using a digital balance. The hydrogel pieces were submerged for a night in $20 \mathrm{ml}$ of absolute ethanol and then weighed after excess ethanol was blotted on the surface. The porosity (\%) was calculated by equation $[19,20]$ :

$$
\text { Porosity }=\frac{\left(M_{2}-M_{1}\right)}{\rho V} \times 100
$$

Where $\left(M_{1}\right)$, and $\left(M_{2}\right)$ are the weight of the hydrogels before and after immersion in absolute ethanol, respectively, while $\rho$ is the actual ethanol density $\left(0.7893 \mathrm{~g} / \mathrm{cm}^{3}\right)$, and $(V)$ is the volume of the swollen hydrogel samples.

\subsubsection{Swelling behaviour}

The hydrogel samples were cut in $2 \mathrm{~cm} \times 2 \mathrm{~cm}$ square and weighed using an analytical balance and were then placed into a $50 \mathrm{~mL}$ centrifuge tube and filled with $40 \mathrm{~mL}$ phosphate-buffered saline solution (PBS) at $37^{\circ} \mathrm{C}$ with $\mathrm{pH}$ 7.4. After that, the pieces of hydrogels were taken from the tube to dry them with filter paper and weighed at different period was $(10,20,30,40,50,60,1440$ minutes). The swelling ratio was determined using the following equation $[21,22]$ :

$$
\text { Swelling }(\%)=\left[\frac{W_{w}-W_{D}}{W_{D}}\right] \times 100 \%
$$

Where $W_{D}$ and $W_{w}$ are the initial weight and the weight at various swelling times of the cut pieces of hydrogel samples, respectively.

\subsubsection{Tensile Testing of KS-Hydrogel Films}

The tensile test was conducted using a texture analyzer (CT3-1000, made in the United States of America), as illustrated in Figure 3.6. The hydrogels were prepared at a speed of $1 \mathrm{~mm} / \mathrm{min}$ with a length of $40 \mathrm{~mm}$, a depth of $1 \mathrm{~mm}$, and a width of $15 \mathrm{~mm}$. For each hydrogel film, the stress ( $(\varepsilon)$-strain (E) curve was determined using the bellow equations [23]:

$$
\varepsilon=\frac{\Delta l}{l}
$$

Where $\Delta l$ and $l$ are the value of the change in length and the initial length, respectively.

$$
E=\frac{\sigma}{\varepsilon}
$$

Where $\sigma$ and $\varepsilon$ represent the initial mass and the mass at various points in time, respectively.

$$
\sigma=\frac{F}{A}
$$

Where $F$ and $A$ denote the applied force and cross-sectional area (mm2), respectively.

2.4.6. Keratin release experiments 
Keratin release from prepared hydrogels was studied using the dialysis technique in tubing cellulose. The preservative of dialysis bag was removed by sacked in $\mathrm{ddH}_{2} \mathrm{O}$ for $12 \mathrm{~h}$ at room temperature. The hydrogel samples have been cut to small sizes $(2 \mathrm{~cm} \times 2 \mathrm{~cm})$ and then placed in a dialysis bag and closed tightly by both sides using plastic clips then placed into a $50 \mathrm{ml}$ tube and filled with $40 \mathrm{ml}$ of $\mathrm{PBS}$ and $\mathrm{pH} 7.4$ at $37^{\circ} \mathrm{C}$. At regular time intervals $(1,2,4,6,12,24,48,72$, and $96 \mathrm{~h})$ was the measured quantity of released keratin from the hydrogels, $2 \mathrm{ml}$ of the medium was withdrawn, replaced with the equivalent volume of fresh PBS and examined for the released keratin by the UV-VIS spectrophotometer (Shimadzu 1800, Japan) [24]. The keratin absorbance was read at $\lambda 246 \mathrm{~nm}$.

2.4.7. Mathematical Modelling of released keratin from hydrogel samples

The release of the keratin was evaluated using kinetic models to study the practical mechanisms of keratin release. The experimental models were applied in four as shown in Table 2. $[25,26]$

Table 2. Equations models to evaluate the release of keratin.

\begin{tabular}{cl}
\hline Model & \multicolumn{1}{c}{ Formula } \\
\hline Zero-order & $C=K_{t}$ \\
First-order & $\operatorname{Ln} C=K_{t}$ \\
Higuchi square root & $C=K_{t}^{0.5}$ \\
Korsmeyer-Peppas & $C=K_{t}^{n}$ \\
\hline
\end{tabular}

\section{Results}

\subsection{FTIR}

FTIR is an effective tool for analyzing the structural bonding of compounds used in KS-hydrogel films, synthesizes and determining their functional groups. The compounds keratin, PVA, PVP, and starch, as well as their functional groups, were defined in five different formulations of KS-hydrogel films' different formulations: KS30, KS40, KS50, KS60, and KS70 films using FTIR spectra with a wavelength range of $500-4000 \mathrm{~cm}^{-1}$ as illustrated in Figure 4.1 and Table. 4.1. According to previous researches, FTIR analysis confirmed the presence of $B$-keratin protein in five different formulations of KS-hydrogel films with their peak assignment $[27,28]$.

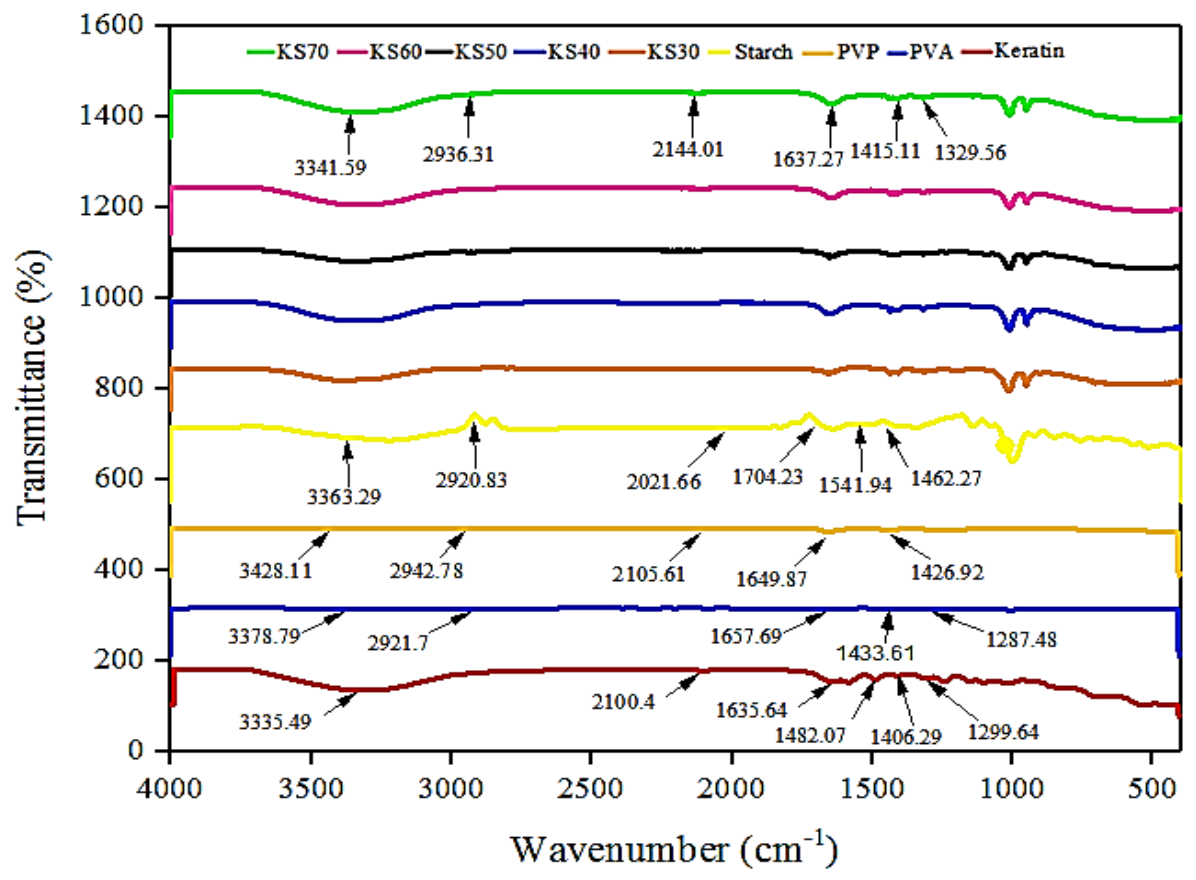


Figure 1. FTIR spectra of KS-hydrogel films (KS30, KS40, KS50, KS60, and KS70), keratin, PVA, PVP, and starch.

Table 3. FTIR peak assignments of the functional group for PVA/PVP/starch/ keratin hydrogel films.

\subsection{Scanning Electron Microscopy (SEM)}

Scanning electron microscopy (SEM) was used to examine the surface morphology for five different formulations of KS-hydrogel films: KS30, KS40, KS50, KS60, and KS70. As illustrated in Figure 2., the top of the KS-hydrogel demonstrated homogeneous network structure, micro-rough surfaces, and micropore architecture. Furthermore, it was observed that the top of KS-30 has the highest micropore architecture compared with other KS- hydrogel samples due to that their keratin concentration was different where the pores decrease gradually with an increase in the keratin content. On the other hand, the appearance of the internal porous network of the hydrogel surfaces that due to its high content of water $[29,30]$. This result was supported the FTIR result that confirms the existence of keratin on the five samples of hydrogel films.

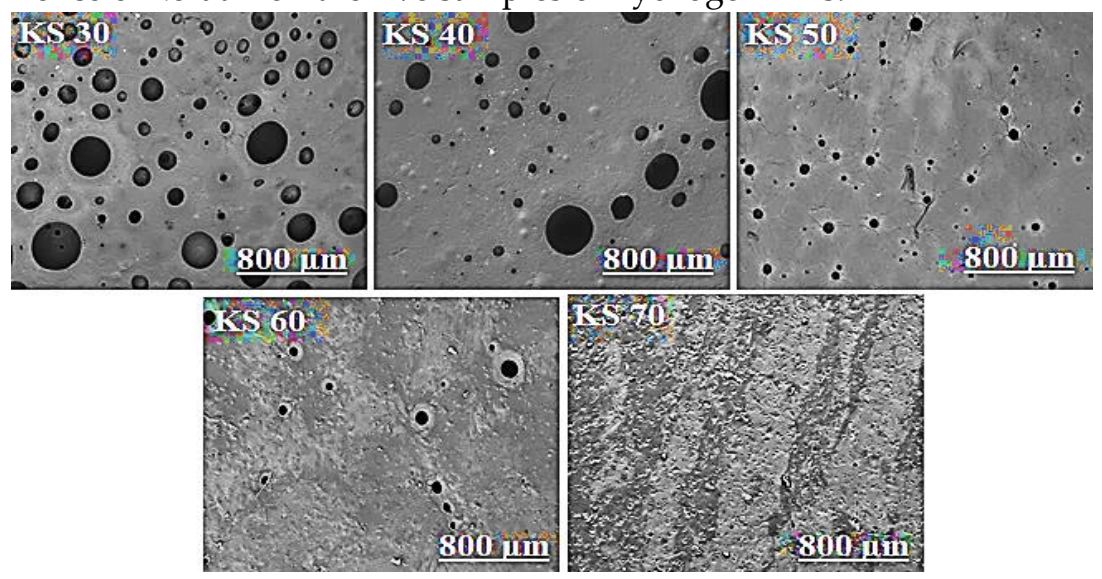

Figure 2. SEM images of KS-hydrogel films (KS30, KS40, KS50, KS60, and KS70). 


\begin{tabular}{|c|c|c|c|c|c|c|c|c|c|}
\hline \multirow[t]{2}{*}{ Assignment } & \multicolumn{9}{|c|}{ Wavenumber $\left(\mathrm{cm}^{-1}\right)$} \\
\hline & Keratin & Starch & $P V A$ & $P V P$ & KS30 & $K S 40$ & KS50 & KS60 & KS70 \\
\hline $\begin{array}{l}\mathrm{O}-\mathrm{H}, \mathrm{N}-\mathrm{H} \\
\text { stretching (Amide } \\
\text { A) }\end{array}$ & 3335.49 & 3363.2 & 3378.7 & 3428.1 & 3341.6 & 3296.5 & 3353.5 & 3466.9 & 3331.2 \\
\hline $\mathrm{C}-\mathrm{H}$ stretching & - & 2920.8 & 2921.7 & 2942.7 & 2936.3 & 2934.6 & 2938.8 & 2935.3 & 2932.7 \\
\hline$C=C$ stretching & 2100.4 & 2021.6 & - & 2105.6 & 2144.0 & 2103.0 & 2116.6 & 2154.4 & 2160.5 \\
\hline $\begin{array}{l}C=O \text { stretching } \\
\text { (Amide I) }\end{array}$ & 1635.6 & 1704.2 & 1657.6 & 1649.9 & 1637.2 & 1640.3 & 1635.3 & 1639.6 & 1638.1 \\
\hline $\mathrm{CH}_{2}$ bending & - & 1462.3 & 1433.6 & 1426.9 & 1415.1 & 1422.9 & 1427.2 & 1432.1 & 1433.6 \\
\hline $\begin{array}{l}\mathrm{C}-\mathrm{N} \text { stretching, } N- \\
\mathrm{H} \text { bending (Amide } \\
\text { III) }\end{array}$ & 1299.6 & - & 1287.4 & - & 1329.6 & 1319.3 & 1321.3 & 1326.7 & 1324.9 \\
\hline
\end{tabular}




\subsection{Porosity Measurement of KS-Hydrogel films}

The interconnected porous structure of the hydrogel can be evaluated by porosity property [31]. The porosities of KS-hydrogel formulations: KS30, KS40, KS50, KS60, and KS70 were using the solvent substitution technique. The porosity ratio of KS-hydrogel films (KS30, KS40, KS50, KS60, and KS70) was obtained, as shown in Figure 3. The results of porosity \% values were recorded at $72.85 \%$ for KS 30, $67.5 \%$ for KS40, $57.77 \%$ for KS50, $44.85 \%$ for KS 60, and 33.57\% for KS70. The porosity of KS-30 hydrogel was increased with a relatively low Interconnecting and high porous structure due to its high-water content. On the other hand, the porosity decreased with a relatively high Interconnecting and low porous structure due to increasing keratin content into the KS- 70 hydrogel. Hydrogel film with high interconnected pore morphology is aided in the absorption of tissue fluid and wound exudate in vivo [29,30].

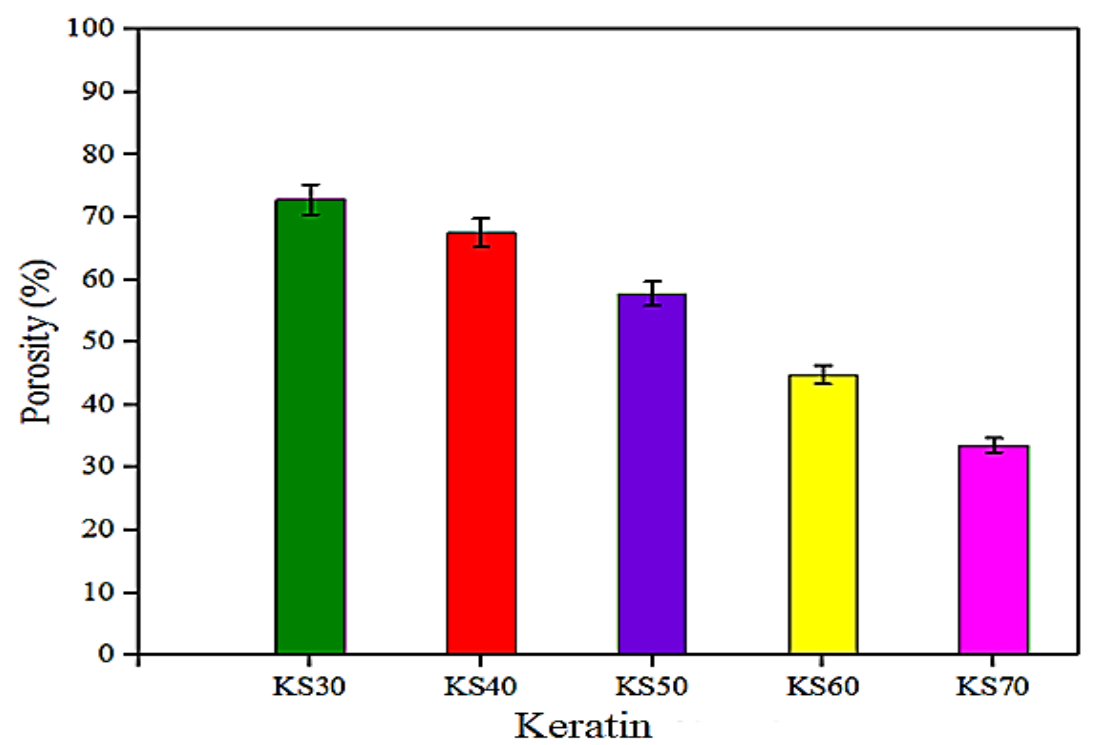

Figure 3. The porosity (\%) of KS-hydrogel films (KS30, KS40, KS50, KS60, and KS70).

\subsection{Swelling Behaviour of KS-Hydrogel Films}

The swelling ratio is used to determine the crosslinking density of the hydrogel structure. The swelling mass ratio was studied by varying the concentration of keratin from 30 to $70 \mathrm{ml}$ and $37^{\circ} \mathrm{C}$ in phosphate-buffered saline (PBS) of KS-hydrogel films. Figure 4 . shows the swelling ratio of the KS-hydrogels. The graph obtained from the chart that with the increase in the keratin content, the swelling ratio decreased, as demonstrated from $59.29 \%$ for KS30 to $53.26 \%$ for KS40, $45.19 \%$ for KS50, 37.87\% for KS60 and 30.66\% for KS70 after $1440 \mathrm{~min}$. Due to the increased crosslinking density between network structures caused by the high keratin content, the swelling ratio of KS-hydrogel films decreased $[30,32]$. 


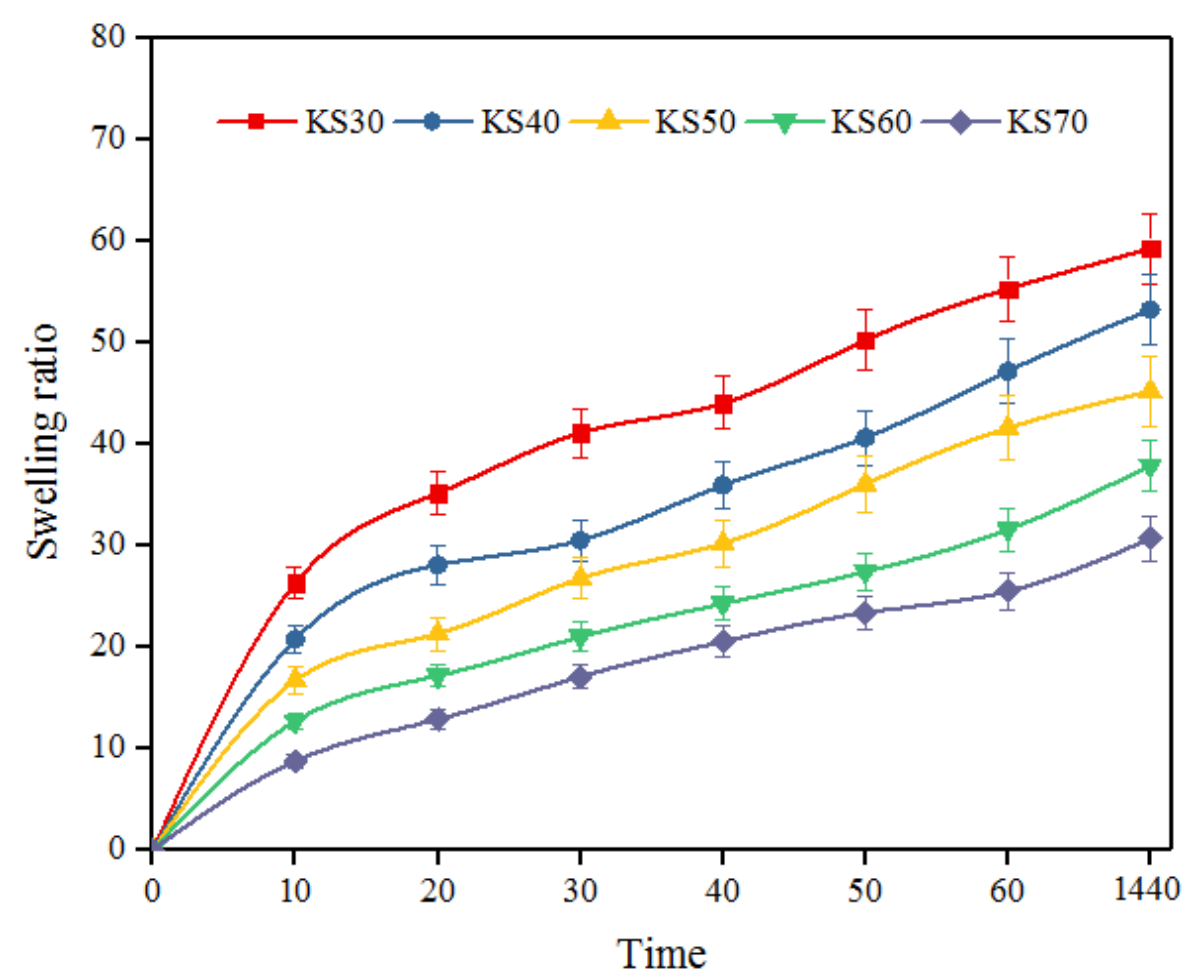

Figure 4. The swelling (\%) of KS-hydrogel films (KS30 KS40, KS50, KS60, and KS70).

\subsection{Tensile Testing of KS-Hydrogel Films}

The tensile test has illustrated the effect of keratin ratio on the KS-hydrogel films (KS30, KS40, KS50, KS60, and KS70 ) mechanical properties, as were plotted in Figure 5. The ultimate strain and stress were calculated from the point of failure. KS-hydrogels point of failure was obtained from the curve at $43.16 \%$ for KS30, 54.04\% for KS40, 58.73\% for KS50, and $69.17 \%$ KS60, and 75\% for KS70. The stress-strain curves of KS-hydrogels show that the increased keratin ratio introduced more interpolymer hydrogen bonds, which improved the hydrogels mechanical strength but with high hardness affected the gel structure [33,34]. 


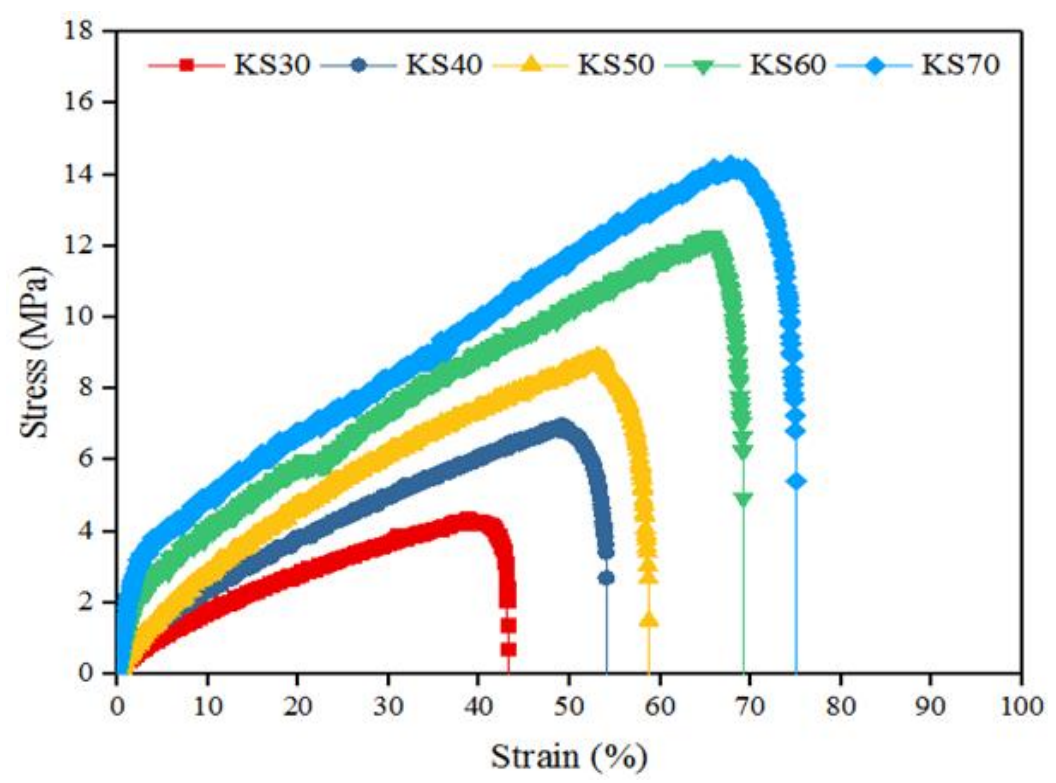

Figure 5. Tensile test of KS-hydrogel films (KS30, KS40, KS50, KS60, and KS70).

\subsection{Keratin Release Ratio and Kinetic Behaviour of KS-Hydrogel Films}

In this section, keratin release and its kinetic were evaluated from KS-hydrogel samples as described below.

\subsubsection{The UV-VIS Absorption Spectra of The Keratin}

The absorbance vs wavelength of the keratin protein was determined using a UV-Vis spectrophotometer. The $40 \mathrm{mg}$ keratin powder was dissolved in $5 \mathrm{~mL}$ PBS (pH 7.4) in a glass flask beaker and stirred for 10 minutes at $37^{\circ} \mathrm{C}$ until the keratin was completely dissolved. As a result, the intensity was $\lambda=246 \mathrm{~nm}$, as shown in Figure 6.

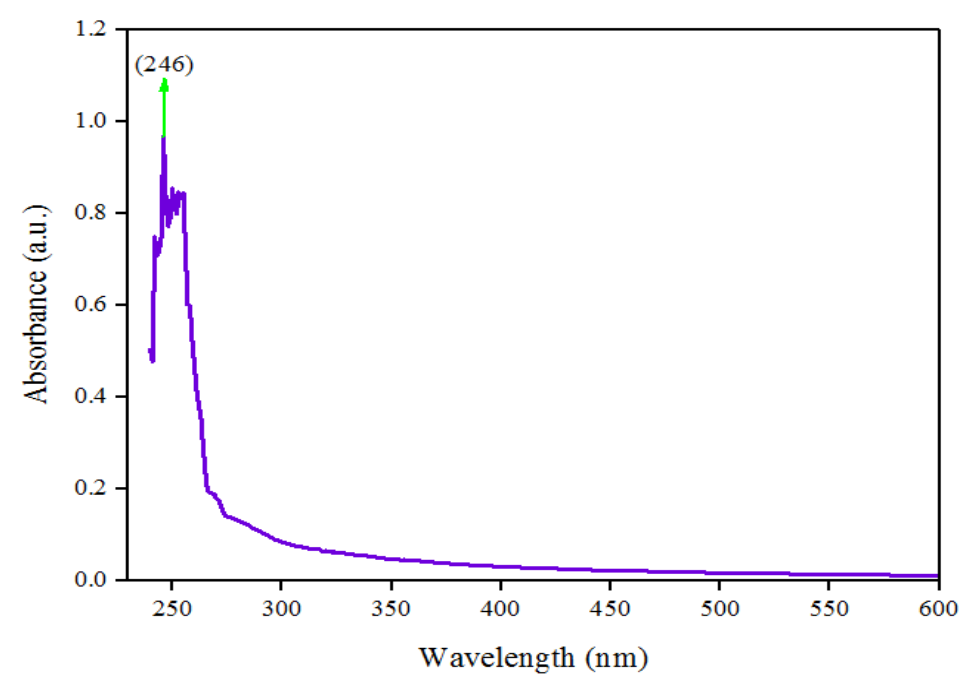

Figure 6. The absorbance (au) vs Wavelength $(\mathrm{nm})$ of keratin into PBS solution.

3.6.2. In Vitro Keratin Release Experiment

The release experiments were conducted to study the cumulative release ratio of the different keratin protein contents at a selected time from the KS-hydrogel films. The 
capability of hydrogels as keratin carriers was evaluated by determining their release behaviour in phosphate-buffered solution (PBS) at $37^{\circ} \mathrm{C}$.

Subsequently, the cumulative keratin release ratio was detected by a UV spectrometer. The cumulative release ratio was obtained from the curve at $67.22 \%$ in $\mathrm{KS} 30,78.2 \%$ in KS40, 83.88\% in KS50, 90.21\% in KS60 and, 95.72\% in KS70, after 96 hr as seen in Figure 7. It was observed from the results that the cumulative keratin release ratio increased as the keratin concentration in hydrogel films increased, indicating that a higher keratin concentration increases the release rate [35]. The observation confirmed the results of the swelling rate and porosity measurement.

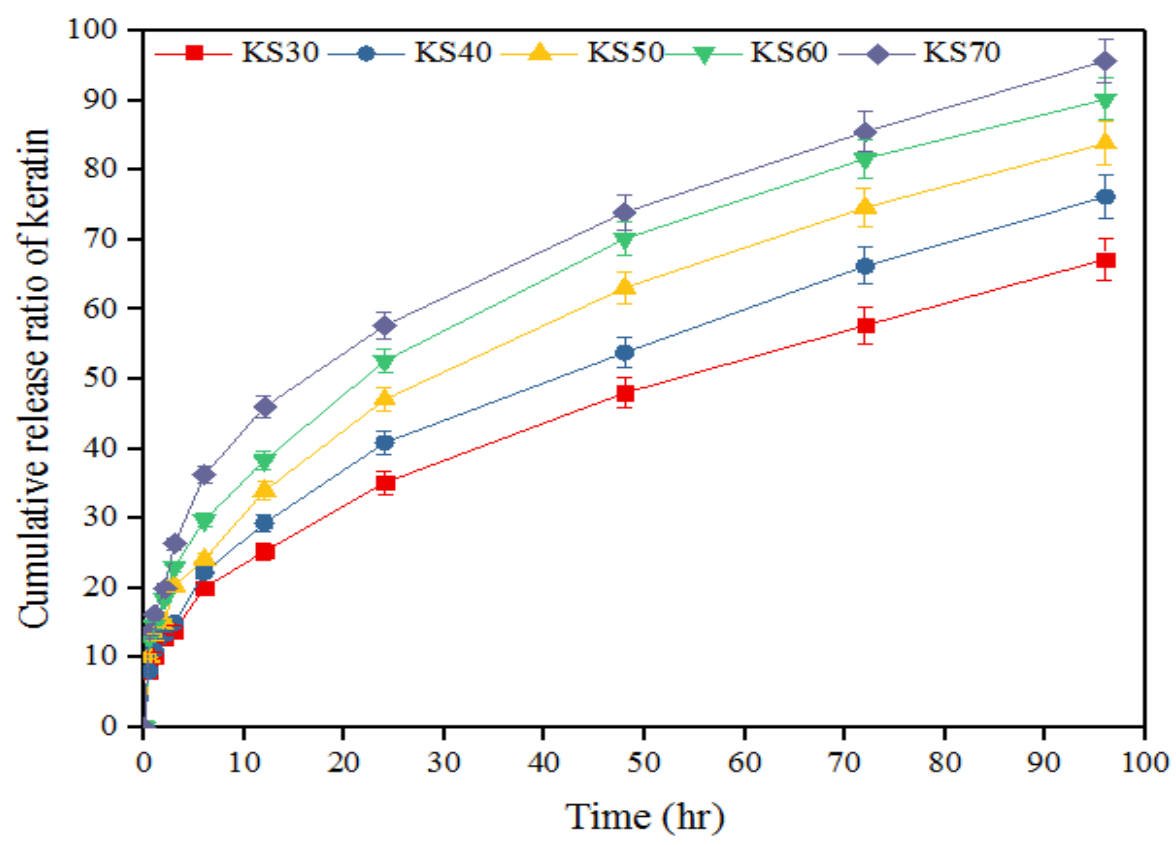

Figure 7. The percentage of cumulative of keratin release form the hydrogel samples.

\subsubsection{Experimental modelling of released keratin from hydrogels}

The release kinetic data of keratin from the hydrogel samples were taken according to four models are showing in Table $3[25,26]$. The regression coefficient $\left(\mathrm{R}^{2}\right)$ values obtained from the Higuchi square root model are greater than those from other kinetic models. The results revealed that the keratin released from hydrogels follows Higuchi kinetics and results are listed in Table 3.

The diffusion exponent (n) values of keratin release were obtained from the Korsmeyer-Peppas model are between ( 0.5894 and 0.6168 with $n$ value less than 1$)$. According to this model, the keratin was indicated Fickian diffusion (anomalous transported). These results are indicating the keratin release depends on swelling behaviour. 
Table 4. The kinetic release date of keratin from hydrogel samples

\begin{tabular}{|c|c|c|c|c|c|c|c|c|c|}
\hline \multirow{3}{*}{$\begin{array}{l}\text { Formulation } \\
\text { Code }\end{array}$} & \multicolumn{9}{|c|}{ Kinetic models } \\
\hline & \multicolumn{2}{|c|}{ Zero order } & \multicolumn{2}{|c|}{ First order } & \multicolumn{3}{|c|}{ Korsmeyer-peppas } & \multicolumn{2}{|c|}{ Higuchi } \\
\hline & $\mathrm{R}^{2}$ & K & $\mathrm{R}^{2}$ & $\mathrm{~K}$ & $\mathrm{R}^{2}$ & $\mathrm{~K}$ & $\mathrm{n}$ & $\mathrm{R}^{2}$ & $\mathrm{~K}$ \\
\hline KS 30 & 0.8902 & 0.6754 & 0.9536 & -0.0051 & 0.7439 & 0.5955 & 0.5955 & 0.9895 & 7.7074 \\
\hline KS 40 & 0.9166 & 0.7867 & 0.9074 & -0.0056 & 0.7512 & 0.6169 & 0.6168 & 0.9960 & 8.1473 \\
\hline KS 50 & 0.9063 & 0.8447 & 0.9827 & -0.0083 & 0.7063 & 0.6021 & 0.60221 & 0.9900 & 9.2935 \\
\hline KS 60 & 0.8924 & 0.8881 & 0.9905 & -0.0130 & 0.6687 & 0.5894 & 0.5894 & 0.9922 & 8.7817 \\
\hline KS 70 & 0.8318 & 0.9207 & 0.9917 & -0.0135 & 0.6511 & 0.5969 & 0.5969 & 0.9693 & 9.8737 \\
\hline
\end{tabular}

\section{Conclusion}

In conclusion, hydrogels were successfully synthesized using keratin from chicken feathers, PVA, PVP and starch. Overall, results indicate that the PVA/PVP at increasing concentrations decreased the maximum the swelling, porosity and keratin released of hydrogels samples. The keratin release was fitted Higuchi square root model with regression coefficient $\left(R^{2}=0.9922\right)$ due to it is the highest value when compared with other $\left(R^{2}\right)$ numbers of kinetics models. The exponent coefficient $(n)$ indicated Fickian diffusion for all hydrogels synthesized. These results proposed that keratin hydrogels could be applied for potential biomedical applications as effective wound healing Conflicts of Interest: The authors declare no conflict of interest.

\section{References}

1. Konstantakos, S.; Marinopoulou, A.; Papaemmanouil, S.; Emmanouilidou, M.; Karamalaki, M.; Kolothas, E.; Saridou, E.; Papastergiadis, E.; Karageorgiou, V. Preparation of model starch complex hydrogels. Food Hydrocoll. 2019, 96, 365-372.

2. He, J.; Shi, M.; Liang, Y.; Guo, B. Conductive adhesive self-healing nanocomposite hydrogel wound dressing for photothermal therapy of infected full-thickness skin wounds. Chem. Eng. J. 2020, 124888.

3. Wang, Y.; Lu, Y.; Zhang, J.; Hu, X.; Yang, Z.; Guo, Y.; Wang, Y. A synergistic antibacterial effect between terbium ions and reduced graphene oxide in a poly (vinyl alcohol)-alginate hydrogel for treating infected chronic wounds. J. Mater. Chem. $B$ 2019, 7, 538-547.

4. Uman, S.; Dhand, A.; Burdick, J.A. Recent advances in shear-thinning and self-healing hydrogels for biomedical applications. J. Appl. Polym. Sci. 2020, 137, 48668.

5. $\quad$ Peppas, N.A.; Hoffman, A.S. Hydrogels. In Biomaterials science; Elsevier, 2020; pp. 153-166.

6. Bala, M.S.; Chin, K.W.; Alashwal, B.Y.; Gupta, A. Development of wound healing spray from keratin protein. Maejo Int. J. Energy Environ. Commun. 2020, 2, 43-49.

7. Gupta, A.; Alashwal, B.Y.; Bala, M.S.; Ramakrishnan, N. Keratin-based Bioplastic from Chicken Feathers Synthesis, Properties, and Applications. Ind. Appl. Biopolym. their Environ. Impact 2020.

8. Donato, R.K.; Mija, A. Keratin Associations with Synthetic, Biosynthetic and Natural Polymers: An Extensive Review. Polymers (Basel). 2020, 12, 32.

9. Rajabi, M.; Ali, A.; McConnell, M.; Cabral, J. Keratinous materials: Structures and functions in biomedical applications. Mater. Sci. Eng. C 2020, 110612.

10. Alashwal, B.Y.; Gupta, A.; Husain, M.S.B. Characterization of dehydrated keratin protein extracted from chicken feather. IOP Conf. Ser. Mater. Sci. Eng. 2019, 702, 012033, doi:10.1088/1757-899X/702/1/012033.

11. Kumar, A.; Kaur, H. Sprayed in-situ synthesis of polyvinyl alcohol/chitosan loaded silver nanocomposite hydrogel for improved antibacterial effects. Int. J. Biol. Macromol. 2020, 145, 950-964.

12. Spicer, C.D. Hydrogel scaffolds for tissue engineering: the importance of polymer choice. Polym. Chem. 2020.

13. Husain, M.; Gupta, A.; Alashwal, B.; Sharma, S. Synthesis of PVA/PVP Based Hydrogel for Biomedical Applications: A 
Review. Energy Sources, Part A Recover. Util. Environ. Eff. 2018, doi:10.1080/15567036.2018.1495786.

14. Husain, M.S.B.; Gupta, A.; Alashwal, B.Y. Development of keratin based hydrogels for biomedical applications. IOP Conf. Ser. Mater. Sci. Eng. 2019, 702, 012031, doi:10.1088/1757-899X/702/1/012031.

15. Munteanu, S.B.; Vasile, C. Vegetable Additives in Food Packaging Polymeric Materials. Polymers (Basel). 2020, 12, 28.

16. Alashwal, B.Y.; Bala, M.S.; Gupta, A.; Sharma, S.; Mishra, P. Improved properties of keratin-based bioplastic film blended with microcrystalline cellulose: A comparative analysis. J. King Saud Univ. 2019.

17. Xu, Y.; Zhou, G.; Liu, L.; Liu, H.; Liu, H.; Niu, X.; Fan, Y. Synthesis and characterization of high-transparent poly (vinyl alcohol)/poly (vinyl pyrrolidone)(PVA/PVP) hydrogels. In Proceedings of the World Congress on Medical Physics and Biomedical Engineering May 26-31, 2012, Beijing, China; Springer, 2013; pp. 67-70.

18. Hassan, C.M.; Peppas, N.A. Cellular PVA hydrogels produced by freeze/thawing. J. Appl. Polym. Sci. 2000, 76, 2075-2079.

19. Ponrasu, T.; Veerasubramanian, P.K.; Kannan, R.; Gopika, S.; Suguna, L.; Muthuvijayan, V. Morin incorporated polysaccharide-protein (psyllium-keratin) hydrogel scaffolds accelerate diabetic wound healing in Wistar rats. RSC Adv. 2018, 8, 2305-2314.

20. Akalin, G.O.; Pulat, M. Preparation and Characterization of Nanoporous Sodium Carboxymethyl Cellulose Hydrogel Beads. J. Nanomater. 2018, 2018.

21. Yu, Z.Y.; Li, Y.; Feng, Z.P.; Zhang, Z.H.; Li, P.; Chen, Y.; Chen, S.S.; Li, P.W.; Yang, Z.M. Cu+-containing physically crosslinked chitosan hydrogels with shape memory. Express Polym. Lett. 2019, 13, 785-793. Sethi, S.; Kaith, B.S.; Kaur, M.; Sharma, N.; Khullar, S. Study of a cross-linked hydrogel of Karaya gum and Starch as a controlled drug delivery system. J. Biomater. Sci. Polym. Ed. 2019, 30, 1687-1708.

23. Mori, H.; Hara, M. Transparent biocompatible wool keratin film prepared by mechanical compression of porous keratin hydrogel. Mater. Sci. Eng. C 2018, 91, 19-25.

24. de Guzman, R.C.; Rabbany, S.Y. PEG-immobilized keratin for protein drug sequestration and pH-mediated delivery. J. Drug Deliv. 2016, 2016.

25. Singh, B.; Kumar, A. Graft and crosslinked polymerization of polysaccharide gum to form hydrogel wound dressings for drug delivery applications. Carbohydr. Res. 2020, 489, 107949.

26. Ganje, M.; Jafari, S.M.; Tamadon, A.M.; Niakosari, M.; Maghsoudlou, Y. Mathematical and fuzzy modeling of limonene release from amylose nanostructures and evaluation of its release kinetics. Food Hydrocoll. 2019, 95, $186-194$.

27. Ramakrishnan, N.; Sharma, S.; Gupta, A.; Alashwal, B.Y. Keratin based bioplastic film from chicken feathers and its characterization. Int. J. Biol. Macromol. 2018.

28. Wang, Y.; Xie, W. Synthesis of cationic starch with a high degree of substitution in an ionic liquid. Carbohydr. Polym. 2010, 80, 1172-1177.

29. Wang, J.; Hao, S.; Luo, T.; Cheng, Z.; Li, W.; Gao, F.; Guo, T.; Gong, Y.; Wang, B. Feather keratin hydrogel for wound repair: Preparation, healing effect and biocompatibility evaluation. Colloids Surfaces B Biointerfaces 2017, 149, 341-350.

30. Lin, C.; Chen, Y.; Tang, K.; Yang, K.; Cheng, N.; Yu, J. Keratin scaffolds with human adipose stem cells: Physical and biological effects toward wound healing. J. Tissue Eng. Regen. Med. 2019, 13, 1044-1058.

31. Song, X.; Zhu, C.; Fan, D.; Mi, Y.; Li, X.; Fu, R.Z.; Duan, Z.; Wang, Y.; Feng, R.R. A novel human-like collagen hydrogel scaffold with porous structure and sponge-like properties. Polymers (Basel). 2017, 9, 638.

32. Yue, K.; Liu, Y.; Byambaa, B.; Singh, V.; Liu, W.; Li, X.; Sun, Y.; Zhang, Y.S.; Tamayol, A.; Zhang, P. Visible light crosslinkable human hair keratin hydrogels. Bioeng. Transl. Med. 2018, 3, 37-48.

33. Wen, J.; Zhang, X.; Pan, M.; Yuan, J.; Jia, Z.; Zhu, L. A Robust, Tough and Multifunctional Polyurethane/Tannic Acid Hydrogel Fabricated by Physical-Chemical Dual Crosslinking. Polymers (Basel). 2020, 12, 239.

34. Silva, R.; Singh, R.; Sarker, B.; Papageorgiou, D.G.; Juhasz, J.A.; Roether, J.A.; Cicha, I.; Kaschta, J.; Schubert, D.W.; Chrissafis, K. Hybrid hydrogels based on keratin and alginate for tissue engineering. J. Mater. Chem. B 2014, 2, 5441-5451.

35. Paarakh, M.P.; Jose, P.A.; Setty, C.; Christoper, G.P. Release kinetics-concepts and applications. Int. J. Pharm. Res. Tech 2018, 
8, 12-20. 